\title{
A Joint Redundancy and Imperfect Maintenance Strategy Optimization for Multi-State Systems
}

\author{
Yu Liu, Hong-Zhong Huang, Member, IEEE, Zhonglai Wang, Yanfeng Li, and Yuanjian Yang
}

\begin{abstract}
The redundancy allocation problem has been extensively studied with the aim of determining optimal redundancy levels of components at various stages to achieve the required system reliability or availability. In most existing studies, failed elements are assumed to be as good as new after repair, from a failure perspective. Due to deterioration, the repaired element cannot always be restored to a virtually new condition unless replaced with a new element. In this paper, we present an approach of joint redundancy and imperfect maintenance strategy optimization for multi-state systems. Along with determining the optimal redundancy levels, the element replacement strategy under imperfect repair is also optimized simultaneously, so as to reach the desired availability with minimal average expenditure. A generalized imperfect repair model is proposed to characterize the stochastic behavior of multi-state elements (MSEs) after repair, and a replacement policy under which a MSE is replaced once it reaches the pre-determined number of failures is introduced. The cost-repair efficiency relation, which regards the imperfect repair efficiency as a function of assigned repair cost, is put forth to provide a flexibility of assigning repair efforts strategically among MSEs. The benefits of the proposed method compared to the existing ones are demonstrated and verified via an illustrative case study of a three-stage coal transportation system.
\end{abstract}

Index Terms-Imperfect maintenance, maintenance resources allocation, multi-state system, redundancy allocation problem.

\section{ACRONYMS}

MSS multi-state system

MSE multi-state element

MTTF mean time to failure

UGF universal generating function

RAP redundancy allocation problem

i.i.d. $\quad s$-independent and identically distributed

\section{NOTATIONS}

$M \quad$ Number of elements in a MSS

$M_{s u b} \quad$ Number of subsystems in a MSS

Manuscript received April 01, 2012; revised September 05, 2012; accepted January 24, 2013. Date of publication April 30, 2013; date of current version May 29, 2013. This work was supported in part by the National Natural Science Foundation of China under Contract 71101017, the Fundamental Research Funds for the Central Universities under Contract 20110185120014, and the Fundamental Research Funds for the Central Universities under Contract ZYGX2011J084. Associate Editor: G. Levitin.

The authors are with the School of Mechanical, Electronic, and Industrial Engineering, University of Electronic Science and Technology of China, Chengdu, Sichuan 611731, China (e-mail: hzhuang@uestc.edu.cn).

Color versions of one or more of the figures in this paper are available online at http://ieeexplore.ieee.org.

Digital Object Identifier 10.1109/TR.2013.2259193
$V_{i} \quad$ Number of available element versions in subsystem $i$

$k_{l} \quad$ Number of possible states of element $l$

$g_{(l, i)} \quad$ Performance rate of element $l$ in state $i$

$p_{(l, i)}(t) \quad$ Probability of element $l$ in state $i$ at time $t$ in the 1 st repair cycle

$p_{(l, i) \mid j}(t) \quad$ Probability of element $l$ in state $i$ at time $t$ in the 1 st repair cycle with initial state $j$

$\lambda_{(i, j)}^{l} \quad$ Intensity of element $l$ transiting from state $i$ to state $j$ in the 1 st repair cycle

$K_{s} \quad$ Number of possible system states

$g_{s i} \quad$ Performance rate of a MSS in its state $i$

$p_{s i}(t) \quad$ Probability of a MSS in state $i$ at time $t$ in the $1 \mathrm{st}$ repair cycle

$p_{(l, i)}^{n}(t) \quad$ Probability of component $l$ in state $i$ at time $t$ in the $n^{t h}$ repair cycle

$p_{(l, i) \mid j}^{n}(t) \quad$ Probability of component $l$ in state $i$ at time $t$ in the $n^{t h}$ repair cycle with initial state $j$

$w_{j}$

$q j$

The $j^{t h}$ possible user demand level

Probability of user demand level being equal to $w_{j}$

$1(x) \quad$ Unity function: $1(T R U E)=1$, and $1(F A L S E)=0$

$A(W) \quad$ System availability under random user demand level

$\bar{C}_{s} \quad$ Average system expenditure

$\bar{c}_{l}$

$c_{l}^{r}$

$c_{l}^{f}$

Average expenditure of element $l$

Replacement cost for element $l$

Assigned repair cost for each repair action performed on element $l$

Maximum repair cost that can be assigned for element $l$

$\alpha_{l}^{0} \quad$ The best efficiency of imperfect repair that can be achieved when the assigned repair cost is $c_{l}^{0}$ for element $l$

$\alpha_{l} \quad$ Quasi-renewal parameter for the lifetime random variable of element $l$

$\beta_{l} \quad$ Quasi-renewal parameter for the repair time random variable of element $l$ 
$\mathbf{T}^{l}(n) \quad$ Transition probability matrix representing the probability of state which repaired element $l$ is restored to in the $n^{\text {th }}$ repair cycle

$r_{(i, j)}^{l}(n) \quad$ Probability of repaired element $l$ being restored from current state $i$ to state $j$ in the $n^{t h}$ repair cycle

$\mathbf{T}_{1}^{l}(n) \quad$ Transition probability vector representing the probability of repaired element $l$ being restored from state 1 to higher performance states in the $n^{\text {th }}$ repair cycle

$\xi_{l} \quad$ Characteristic constant related to imperfect repair efficiency of element $l$

$\zeta_{(l, i)} \quad$ Characteristic constant related to the transition probability $q_{(i, i)}^{l}$ of element $l$

$X_{(n, i) \mid j}^{l} \quad$ Random time of element $l$ sojourning in state $i$ during the $n^{t h}$ repair cycle with initial state $j$

$X_{n}^{l} \quad$ Random lifetime of element $l$ in the $n^{\text {th }}$ repair cycle

$S_{(n, i)}^{l} \quad$ Random time of element $l$ sojourning in state $i$ during the $n^{\text {th }}$ repair cycle

$Y_{n}^{l} \quad$ Random repair time of element $l$ in the $n^{\text {th }}$ repair cycle

$T_{n}^{l} \quad$ Time duration of the $n^{\text {th }}$ replacement cycle for element $l$

$\mu_{X_{n}^{l}} \quad$ Mean time to failure of element $l$ in the $n^{\text {th }}$ repair cycle

$\mu_{X_{n \mid j}^{l}} \quad$ Mean time to failure of element $l$ in the $n^{\text {th }}$ repair cycle with initial state $j$

$\mu_{Y_{n}^{l}} \quad$ Expected mean time of repairing element $l$ in the $n^{\text {th }}$ repair cycle

$\mu_{S_{(n, i)}^{l}} \quad$ Expected mean time of element $l$ sojourning in state $i$ during the $n^{\text {th }}$ repair cycle

$\mu_{S_{(n, i) \mid j}^{l}} \quad$ Expected mean time of element $l$ sojourning in state $i$ during the $n^{\text {th }}$ repair cycle with initial state $j$

$\mu_{Z_{l}} \quad$ Expected mean time of replacing element $l$

$\bar{p}_{(l, i)} \quad$ Average probability of element $l$ sojourning in state $i$

$\bar{p}_{s i} \quad$ Average probability of entire system sojourning in state $i$

$E(\cdot) \quad$ Expectation operator

\section{INTRODUCTION}

$\mathbf{R}$ EDUNDANCY strategy, as a way to improve the system reliability through incorporating redundant components or subsystems, has been extensively adopted in industry. The redundancy allocation problem (RAP) focuses on choosing a set of components available in the market, and determines optimal redundancy levels of components at various stages to achieve the required system reliability or availability within the physical constraints, e.g. weight, volume etc., or with minimal cost. It is a paramount research topic, and has received increasing attention in these years [1].

The traditional RAP deals with the binary state system model, where components and systems only have two possible states: working perfectly, or completely failed. As seeking the optimal solution in an enumerative way is computationally burdensome, both heuristic algorithms (e.g. branch-and-bound method [2], [3], scaling method [4] etc.) and meta-heuristic algorithms (e.g. such as genetic algorithm [5], ant colony algorithm [6], tabu search [7], annealing simulated algorithm [8], etc.) have been intensively studied to increase the global optimization capacity for RAPs. However, many practical engineering systems, such as nuclear systems, manufacturing systems, computing systems etc., can perform their intended functions at more than two performance rate levels, from a perfect functioning state with a nominal performance rate, to complete failure. These kinds of systems are called multi-state systems (MSSs), and their RAPs have received considerable attention in recent years [9]. The redundancy optimization for multi-state series-parallel systems is first reported in [10]. Heuristic approaches are introduced to solve the resulting RAP in a computationally efficient manner [11], [12]. The reliability-redundancy allocation scheme, in which the target MSS reliability can be achieved via either providing redundancy levels or improving component state distributions, is proposed in [13]. Nevertheless, the aforementioned RAPs only treat the system construction cost (component cost) as the objective or constraint. As mentioned in [14], component cost, by itself, is an inadequate measure of a system's operational effectiveness. Even a system with high reliability might result in a higher total cost of ownership (TCO) which includes component cost, operation cost, as well as maintenance and support cost. To maximize system efficiency from the lifecycle view point, decision-makers should, therefore, take into account the lifecycle cost rather than only the construction cost in the system design phase. Few works have investigated the RAP in conjunction with maintenance strategy. Levitin and Lisnianski [15] formulate a joint redundancy and replacement schedule optimization problem for MSSs, where the element versions, redundancy levels, as well as the replacement intervals are optimized simultaneously. Nourelfath et al. [16], [17] discuss the RAP for MSSs considering limited maintenance repairmen. A joint reliability-redundancy optimization is studied by Tian et al. [18] considering technical and organizational actions which might affect degradations and repair rates of MSEs. However, the aforementioned research is based on the perfect and minimal maintenance assumptions that repaired elements are in either as good as new condition or as bad as old condition, from a reliability point of view. The unrealistic hypothesis has been frequently argued in literature, and many imperfect maintenance models which treat repaired element and systems as in a physical condition somewhere between the two extremes have been proposed in the past decade [19]. The most relevant efforts among them are: $(p, q)$ model (Nakagawa [20]), $(p(t), q(t))$ model (Block et al. [21]), $(p(n, t), q(n, t), s(n, t))$ model (Makis and Jardine [22]), Kijima Type I and II models (Kijima [23], [24]), improvement factor method (Malik [25]), hybrid imperfect model (Lin [26]), geometric process (Lam [27]), and 
quasi-renewal model (Wang and Pham [28], [29]). The commonality and interrelationship among them are investigated in [30]. Nevertheless, most of the imperfect maintenance models deal with binary state elements and systems. To the best of our knowledge, quantifying imperfect maintenance efficiency for elements and systems possessing multiple states is still in infancy, and only a few attempts have been made to date. For example, Levitin et al. [31], and Nahas et al. [32] employ the age reduction model to quantify the improvement of element condition resulting from the preventive maintenance (PM). By using the Kijima type II imperfect maintenance model, Liu and Huang [33] develop a selective maintenance strategy for MSSs with binary state elements. Because these imperfect maintenance models can only be applied to the elements with binary capacity, it becomes infeasible once MSE cannot be categorized or simplified to a binary state element. Soro et al. [34] introduce a series of performance measures for the MSS under minimal and imperfect maintenance action; and one kind of imperfect PM action recovering the MSS from the last acceptable state to a higher but degraded state is modeled via a Markov process. Tan and Raghavan [35] study a system oriented maintenance framework, and introduce a random restoration factor quantifying the imperfect restoration. Some possible errors and misunderstanding associated with their work have been discussed in [36]. To quantify the efficiency of imperfect preventive maintenance for MSEs, Nourelfath et al. [37] put forth a Markov model for preventively maintained MSEs. The MSE which has degraded to its unacceptable state will restore to a better state after a preventive maintenance. Both the redundancy allocation and PM planning are optimized in a concurrent manner to maximize system availability under a budget constraint. An alternative imperfect maintenance model based on the quasi-renewal process of binary state systems has been developed in our recent paper [38]. The key idea behind it is that, even though the repaired element is restored to its best state after repair, it cannot be considered to be in a completely new condition, and the degradation trend accelerates in the next repair cycle. This important phenomena has been extensively observed in many engineered systems [38], but cannot be characterized by existing imperfect maintenance models of MSSs [31], [34], [35], [37]. As an extension of our previous work, a RAP incorporated with element replacement strategy optimization under imperfect repair is formulated in this paper. By releasing the assumption that the repaired element is always recovered to its best state as in our earlier work, a more generalized imperfect repair model for repairable MSEs is also introduced. The repair activity for individual elements will be executed once the element falls into its failure state. With the increase of repair times, the working durations of repaired elements will decrease while the durations for repairing will increase. It is more economically efficient to replace the deteriorated element $l$ with a brand new one after the pre-determined number of failures $N_{l}$ than to continue repairing. This element replacement policy is called policy $N_{l}$ throughout the present paper. Furthermore, it is reasonable to relate the repair efficiency with the assigned repair cost. The more repair resources spent, the better condition the repaired element will restore to. Another contribution of this work compared with the existing literature [31], [32], [37] lies in the introduction of two hypothetical functional relations which

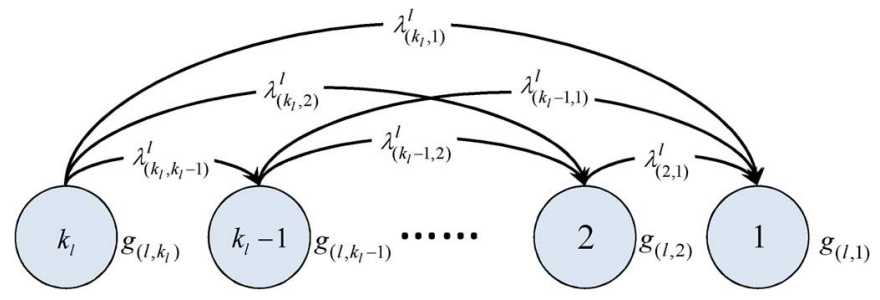

Fig. 1. The state-space diagram of the MSE $l$.

enable us to treat the imperfect repair efficiency as a function of the assigned repair expenditure. This idea is highly desired in practical maintenance management because it provides much more flexibility to the maintenance decision makers. With such flexibility, they can determine not only when to replace a deteriorated element, but also how much repair resources are to be assigned for each repair action, to achieve the required system availability with a minimum expected expenditure per unit time for the entire MSS.

The remainder of this paper is organized as follows. Section II introduces the Markov model of the concerned MSSs in this paper. The proposed generalized imperfect repair model for MSEs is presented in Section III. The hypothetical functions linking the repair efficiency and cost are formulated in Section IV. The formulation of the joint RAP is formulated in Section V. The genetic algorithm (GA) and its implementation on our specific problem are briefly reviewed in Section VI. An illustrative case is presented in Section VII, and it is followed by remarks and closure in Section VIII.

\section{MARKov MOdel FOR MSE Without RePAiR}

The MSS studied in this work is defined as a system having a finite number of states with distinct performance rates [9]. Many engineered systems such as power generating systems, or water pipe systems, can be viewed as MSSs [39], [40], [41]. The performance rate of the entire MSS at any time instant $t$ is a discrete random quantity, and can be completely determined by the performance rates of its components and the system configuration.

Directly constructing a stochastic model for the entire MSS with a great amount of components is intractable due to state explosion. Lisnianski [42] introduces a novel method to integrate the stochastic process model of individual MSE via UGF. In this way, the number of states in the associated stochastic model can be drastically reduced. Following the aforementioned modeling strategy, the stochastic behavior of individual MSE without any repair is modeled by the homogenous Markov process with the assumption that the transition time between any pair of states is exponentially distributed. An example of the state-space diagram of the element $l$ without considering repair activity is shown in Fig. 1. The corresponding Kolmogorov differential equations for element $l$ can be written as [43]

$$
\frac{d p_{(l, i)}(t)}{d t}=\sum_{j=i+1}^{k_{l}} \lambda_{(j, i)}^{l} p_{(l, j)}(t)-p_{(l, i)}(t) \sum_{j=1}^{i-1} \lambda_{(i, j)}^{l} .
$$

If the element starts working from state $j(j>1)$, the initial conditions should be given as $p_{(l, j)}(0)=1, p_{(l, i)}(0)=0$ for $(i \neq j)$, and the state distribution with respect to time $t$ can be obtained via solving this set of differential equations. 


\section{A GENERALIZED IMPERFECT REPAIR MODEL}

Most maintenance models using a renewal process are based on the assumption that maintenance actions are always perfect, and repaired systems are restored to as good as new condition. To model the imperfect maintenance of binary state systems, Wang and Pham [28] introduced the quasi-renewal process to characterize the deteriorating trend of the repaired system. However, the quasi-renewal process has only been successfully applied to model the imperfect repair of binary state systems, and its application to MSS, especially the one concerned in this paper, has heretofore not been completed.

In our recent paper [38], we assume that a MSE, either failed or working, can be recovered to its best state after repair action, but not as good as a brand new one. The state transition intensities increase proportionally after repair. Hence, the pace of the degradation from the best state to a lower state becomes faster in the next repair cycle. This assumption is more realistic and reasonable because, unless replaced, the repair element cannot restore to its initial state due to aging and deterioration, and the degradation process will accelerate with usage. With this kind of consideration, the quasi-renewal process is extended to characterize the deterioration trend of MSEs with respect to the number of repairs as follows.

A set of sequential nonnegative random variables $\left\{X_{(1, i)}^{l}, X_{(2, i)}^{l}, \ldots, X_{(n, i)}^{l}, \ldots\right\}$ is used to represent the time duration of MSE $l$ sojourns in state $i$. Suppose $X_{(1, i)}^{l}=Z_{(1, i)}$, $X_{(2, i)}^{l}=\alpha_{l} \cdot Z_{(2, i)}, X_{(3, i)}^{l}=\alpha_{l}^{2} \cdot Z_{(3, i)}, \ldots\left(0<\alpha_{l}<1\right)$ hold, and random quantities $Z_{(n, i)}, n \in\{1,2, \ldots\}$ are i.i.d. The sequence of random variables $\left\{X_{(1, i)}^{l}, X_{(2, i)}^{l}, \ldots, X_{(n, i)}^{l}, \ldots\right\}$ is said to be monotonically decreasing random variables. Furthermore, the counting process $\left\{N^{l}(t), t \geq 0\right\}$ representing the cumulative times that MSE $l$ falls into the worst state 1 from the best state $k_{l}$ forms a decreasing quasi-renewal process once the state transition intensities increase proportionally. Thus, if the state probability of a brand new MSE in the first repair cycle is denoted as $p_{(l, i)}(t), i \in\left\{1,2, \ldots, k_{l}\right\}$, one can derive the state probability of the MSE in the $n$ repair cycle as $p_{(l, i)}\left(\alpha_{l}^{1-n} t\right)$. Obviously, these relations reflect the proportionally increasing trend of the state transition intensity with respect to the number of repairs. Therefore, the expected time of element $l$ sojourning in state $i$ during the $n^{t h}$ repair cycle is given by

$$
\begin{aligned}
E\left(S_{(n, i)}^{l}\right) & =\int_{0}^{+\infty} p_{(l, i)}^{n}(t) d t=\int_{0}^{+\infty} p_{(l, i)}\left(\alpha_{l}^{1-n} t\right) d t \\
& =\alpha_{l}^{n-1} \cdot \mu_{S_{(1, i)}^{l}},
\end{aligned}
$$

where $\mu_{S_{(1, i)}^{l}}$ is the expected time of element $l$ staying at state $i$ in the first repair cycle. If the element state 1 is regarded as a failure state, then the mean time to failure (MTTF) in the $n^{\text {th }}$ repair cycle is given by

$$
\begin{aligned}
E\left(X_{n}^{l}\right) & =\int_{0}^{+\infty} \sum_{i=2}^{k_{l}} p_{(l, i)}^{n}(t) d t=\int_{0}^{+\infty} \sum_{i=2}^{k_{l}} p_{(l, i)}\left(\alpha_{l}^{1-n} t\right) d t \\
& =\alpha_{l}^{n-1} \cdot \mu_{X_{1}^{l}}
\end{aligned}
$$

where $\mu_{X_{1}^{l}}$ is the MTTF of element $l$ in the first repair cycle.

However, in our preliminary work [38], the basic assumption that any element after repair can recover to its best state is somewhat restrictive. A more generalized imperfect repair model is to account for the case where repaired elements are not necessarily always being restored to their best state but might also be recovered to any intermediate states with a higher performance rate. The new imperfect repair model is applicable to many practical cases. For instance, the undetected fault may still exist after insufficient inspection and repair. Thereby, the repaired element can only be recovered to its intermediate states with degraded performance [44], [45]. Another example is the situation where some of the aging and deteriorations of elements are non-repairable (e.g. power systems [45] etc.); repaired elements have a degraded performance rate after repair [34]. The probability that a repaired element returns to one of the higher performance states is quantified via a transition probability matrix with respect to the number of repair cycles $n$, which is defined as (4) shown at the bottom of the page. where $r_{(i, j)}^{l}(n)$ $\left(i, j \in\left\{1,2, \ldots, k_{l}\right\}\right.$, and $r_{(i, j)}^{l}(n)=0$ for $\left.j \leq i\right)$ is the probability that repaired element $l$ is restored from current state $i$ to the higher performance state $j$ in the $n^{\text {th }}$ repair cycle, and it satisfies the condition $\sum_{j=i+1}^{k_{l}} r_{(i, j)}^{l}(n)=1$. This condition holds because, after repair, the repaired element can always be in one and only one higher performance state from $i+1$ to $k_{l}$, and it composes the complete group of mutually exclusive events. In general, the transition probability can be estimated and inferred via maintenance and operation records, as well as the knowledge from experts. It is also worth mentioning that, with the number of repair cycles $n$ increasing, the transition probability from the repaired state to the higher state decreases while the probability to the lower state increases. In other words, it becomes harder to restore an element to its better states as the number of repairs increases. Though this behavior is important, and can be widely observed in engineering practice, it cannot be characterized by many imperfect maintenance models reported in literature [31], [32], [37].

$$
\mathbf{T}^{l}(n)=\begin{gathered}
\text { state } \\
1 \\
2 \\
\vdots \\
k_{l}-2 \\
k_{l}-1
\end{gathered}\left[\begin{array}{ccccc}
0 & r_{(1,2)}^{l}(n) & \cdots & r_{\left(1, k_{l}-1\right)}^{k_{l}-1} & k^{k}(n) \\
0 & 0 & \cdots & r_{\left(2, k_{l}-1\right)}^{l}(n) & r_{\left(2, k_{l}\right)}^{l}(n) \\
\vdots & \vdots & \ddots & \vdots & \vdots \\
0 & 0 & \cdots & r_{\left(k_{l}-2, k_{l}-1\right)}^{l}(n) & r_{\left(k_{l}-2, k_{l}\right)}^{l}(n) \\
0 & 0 & \cdots & 0 & 1
\end{array}\right]
$$


In this paper, we only demonstrate our method via a specific scenario where the worst element state is treated as the failure state of each element, and the repair action is triggered once an element fails. This sort of maintenance strategy has been adopted in engineering practice when conducting any maintenance activity is expensive, and would incur too much production lost. For example, for some sophisticated manufacturing systems, performing preventive maintenance at degraded states of a product line (can be viewed as a MSE) requires turning off this product line, which may incur expensive inspection and disassembly costs, as well as a huge production lost. Some infrastructure systems and facilities may also face the same issue. For instance, maintaining buried pipe systems often incurs much additional cost from digging trenches. In all of these cases, it is much more economically efficient to perform maintenance whenever elements fall into an unacceptable or failure state. In this scenario, the probability matrix of element $l$ restoring to the higher performance states after repair can be abstracted from the first row of $\mathbf{T}^{l}(n)$, and is denoted as $\mathbf{T}_{1}^{l}(n)=\left[r_{(1,2)}^{l}(n), \ldots, r_{\left(1, k_{l}-1\right)}^{l}(n), r_{\left(1, k_{l}\right)}^{l}(n)\right]$. Because it is possible for the repaired element to start working while in the degraded states (i.e. state 2 to state $k_{l}-1$ ) after repair, one needs to evaluate the state distribution in these situations. Resorting to the Markov model introduced in Section 2.2, the state distribution of element $l$ with initial state $j, j \in\left\{2,3, \ldots, k_{l}-1\right\}$, can be obtained through solving (1) under the condition $p_{(l, j)}(0)=1$ and $p_{(l, i)}(0)=0$ for $(i \neq j)$. To make the notation clearer, in the rest of this paper, we use $p_{(l, i) \mid j}(t)$ to represent the probability of element $l$ staying at state $i$ in the first repair cycle with initial state $j$, and $p_{(l, i) \mid j}^{n}(t)$ to represent the probability of element $l$ staying at state $i$ in the $n^{\text {th }}$ repair cycle with initial state $j$. Therefore, based on the aforementioned imperfect repair model, a set of sequential nonnegative random variables $\left\{X_{(1, i) \mid j}^{l}, X_{(2, i) \mid j}^{l}, \ldots, X_{(n, i) \mid j}^{l}, \ldots\right\}$, where $X_{(n, i) \mid j}^{l}\left(i, j \in\left\{1,2, \ldots, k_{l}\right\}, n \in\{1,2, \ldots\}, j \geq i\right)$ denotes the random time that element $l$ sojourns in state $i$ during the $n^{\text {th }}$ repair cycle with initial state $j$. In the same fashion, if one assumes the relations $X_{(1, i) \mid j}^{l}=Z_{(1, i) \mid j}, X_{(2, i) \mid j}^{l}=\alpha_{l} \cdot Z_{(2, i) \mid j}$, $X_{(3, i) \mid j}^{l}=\alpha_{l}^{2} \cdot Z_{(3, i) \mid j}, \ldots$ hold, where random quantities $Z_{(n, i) \mid j}, n \in\{1,2, \ldots\}$ are i.i.d., the sequence of random variables $\left\{X_{(1, i) \mid j}^{l}, X_{(2, i) \mid j}^{l}, \ldots, X_{(n, i) \mid j}^{l}, \ldots\right\}$ also has the monotonically decreasing character, and the probability $p_{(l, i) \mid j}^{n}(t)$ can be derived as $p_{(l, i) \mid j}\left(\alpha_{l}^{1-n} t\right)$. Accordingly, the time duration of element $l$ sojourning in state $i$ during the $n^{\text {th }}$ repair cycle is given by

$$
S_{(n, i)}^{l}=\sum_{j=i}^{k_{l}} r_{(1, j)}^{l}(n-1) \cdot X_{(n, i) \mid j}^{l}
$$

The associate state probability is expressed as

$$
p_{(l, i)}^{n}(t)=\sum_{j=i}^{k_{l}} r_{(1, j)}^{l}(n-1) \cdot p_{(l, i) \mid j}\left(\alpha_{l}^{1-n} t\right) .
$$

In the same fashion as (2), the expectation of $S_{(n, i)}^{l}$ can be written as

$$
E\left(S_{(n, i)}^{l}\right)=\int_{0}^{+\infty} p_{(l, i)}^{n}(t) d t=\alpha_{l}^{n-1} \sum_{j=i}^{k_{l}} r_{(1, j)}^{l}(n-1) \cdot \mu_{S_{(1, i) \mid j}^{l}},
$$

where $\mu_{S_{(1, i) \mid j}^{l}}$ is the expected time of the element staying at state $i$ in the first repair cycle with initial state $j$. Because the element state 1 is viewed as the failure state, one can derive the MTTF of the $n^{\text {th }}$ repair cycle, analogous with (3), as

$$
E\left(X_{n}^{l}\right)=\int_{0}^{+\infty} \sum_{i=2}^{k_{l}} p_{(l, i)}^{n}(t) d t=\alpha_{l}^{n-1} \sum_{j=2}^{k_{l}} r_{(1, j)}^{l}(n-1) \cdot \mu_{X_{1 \mid j}^{l}},
$$

where $\mu_{X_{1 \mid j}^{l}}$ is the MTTF of element $l$ in the first repair cycle with initial state $j$.

On the other hand, the duration of each repair activity follows the monotonically increasing trend with respect to the number of repairs, and it is represented by an increasing quasi-renewal process with the parameter $\beta_{l}>1$. The successive repair durations form a sequence of nonnegative random variables $\left\{Y_{1}^{l}, Y_{2}^{l}, \ldots, Y_{n}^{l}, \ldots\right\}$. The expected repair time for element $l$ in the $n^{\text {th }}$ repair cycle is given by

$$
E\left(Y_{n}^{l}\right)=\beta_{l}^{n-1} E\left(Y_{1}^{l}\right)=\beta_{l}^{n-1} \cdot \mu_{Y_{1}^{l}},
$$

where $\mu_{Y^{l}}$ is the mean time of repairing element $l$ in the first repair cycle.

The replacement policy being considered for each individual element is called policy $N_{l}$, in which the element is replaced by an identical brand new one once its number of failures reaches the pre-determined value $N_{l}$. Let $T_{m}^{l}(m \geq 1)$ be the time duration of the $m^{\text {th }}$ replacement cycle. Apparently, $\left\{T_{1}^{l}, T_{2}^{l}, \ldots, T_{m}^{l}, \ldots\right\}$ forms a renewal process. We use random variable $Z_{l}$ denoting the time spent on replacement action, and $\mu_{Z_{l}}$ denoting its expected mean value. Thus, the average probability $\bar{p}_{(l, i)}$ that the element $l$ sojourns in state $i$ under policy $N_{l}$ can be computed by

$$
\begin{aligned}
\bar{p}_{l, i} & =\frac{\text { mean time staying at state } i \text { in a renewal cycle }}{\text { mean length of a renewal cycle }} \\
& =\frac{E\left(S_{i}^{l}\left(N_{l}\right)\right)}{E\left(Y_{l}\left(N_{l}-1\right)\right)+E\left(X_{l}\left(N_{l}\right)\right)+E\left(Z_{l}\right)}
\end{aligned}
$$

where

$$
\begin{aligned}
& E\left(Z_{l}\right)= \mu_{Z_{l}} \\
& E\left(X_{l}\left(N_{l}\right)\right)=E\left(\sum_{n=1}^{N_{l}} X_{n}^{l}\right)=\sum_{n=1}^{N_{l}} \sum_{j=2}^{k_{l}} \alpha_{l}^{n-1} \\
& \cdot r_{(1, j)}^{l}(n-1) \cdot \mu_{X_{1 \mid j}^{l}} \\
& E\left(Y_{l}\left(N_{l}-1\right)\right)=E\left(\sum_{n=1}^{N_{l}-1} Y_{n}^{l}\right)=\sum_{n=1}^{N_{l}-1} \beta_{l}^{n-1} \cdot \mu_{Y_{1}^{l}} \\
& E\left(S_{i}^{l}\left(N_{l}\right)\right)=E\left(\sum_{n=1}^{N_{l}} S_{(n, i)}^{l}\right)=\sum_{n=1}^{N_{l}} \sum_{j=i}^{k_{l}} \alpha_{l}^{n-1} \\
& \cdot r_{(1, j)}^{l}(n-1) \cdot \mu_{S_{(1, i) \mid j}^{l}}
\end{aligned}
$$

\section{REPAir EFficiency Versus Assigned Repair Cost}

To reflect the relation between improvement from repair and its related cost, Lie and Chuan [46] implement a set of functions to build accurate functional relationships between the ratio of PM cost to preventive replacement cost, and the system improvement after PM. Malik [47] points out that the maintenance 
improvement can be approximately inferred via expert judgment. Intuitively, if the spent repair cost approaches zero, the improvement will approach zero also; oppositely, if the repair cost approaches the maximum value, the repaired system would approach the as good as new condition [33]. Based on this relation, and the imperfect repair model proposed in this paper, the repair expenditure has twofold impacts on the repair efficiency. As more resources are spent, the deteriorating trend of the repaired element will become lower in the next repair cycle, and the element will have a greater probability of recovering to a higher state after repair. In the ensuing paragraphs, two hypothetical functions linking the assigned repair cost to its impact on the repaired element are proposed to mathematically describe the exact relationship.

The imperfect repair parameter $\alpha_{l}$ in our proposed model is defined as a power function of the assigned repair cost as follows

$$
\alpha_{l}=\alpha_{l}^{0}\left(\frac{c_{l}^{f}}{c_{l}^{0}}\right)^{\frac{1}{\xi_{l}}}
$$

where $0<c_{l}^{f} \leq c_{l}^{0}, \xi_{l}>0 . \alpha_{l}^{0}$ and $\xi_{l}$ can be estimated via experts' knowledge, as well as the operation and maintenance records from experiments and field use. It is obvious that, with a higher $\xi_{l}$, the repair is more effective even if the expenditure is low, corresponding to the situation where the element would restore to a better condition via fixing a few parts and modules. On the other hand, if $\xi_{l}$ is lower than 1.0, it can be found that repair action is less effective, particularly with low expenditure. It corresponds to the scenario where the element repair efficiency is not significant unless most of the parts and modules are being repaired.

As this paper only considers the scenario where an element is repaired once it falls into the worst state, we can only define $\mathbf{T}_{1}^{l}(n)$ instead of $\mathbf{T}^{l}(n)$. Hence, $\mathbf{T}_{1}^{l}(n)$ is written as

$$
\mathbf{T}_{1}^{l}(n)=\mathbf{I} \times \mathbf{Q}_{l}^{n},
$$

where $\mathbf{I}=[0, \ldots, 0,1]$ is a $1 \times\left(k_{l}-1\right)$ vector, and

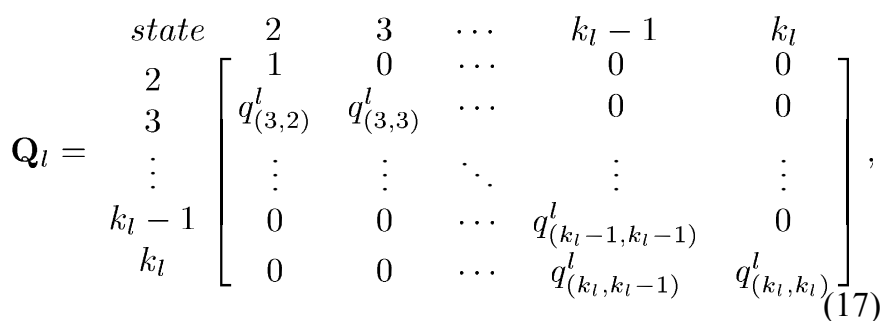

where $q_{(i, j)}^{l}\left(i, j \in\left\{2, \ldots, k_{l}\right\}, q_{(i, j)}^{l} \neq 0\right.$ for $i-1 \leq j \leq$ $i)$ represents the one-step transition probability that the state, which the element is being restored to, transits from state $i$ to state $j$, and it satisfies $q_{(i, i)}^{l}+q_{(i, i-1)}^{l}=1$. To link the $\mathbf{T}_{1}^{l}(n)$ with the assigned repair cost, a hypothetical function defining $q_{(i, i)}^{l}$ as a power function of the assigned repair $c_{l}^{f}$ is given as

$$
q_{(i, i)}^{l}=\left(\frac{c_{l}^{f}}{c_{l}^{0}}\right)^{\frac{1}{\zeta_{(l, i)}}}
$$

where the characteristic parameter $\zeta_{(l, i)}\left(\zeta_{(l, i)}>0\right.$ for $\left.i>2\right)$ is also estimated via expert's knowledge, and historical maintenance records.

\section{MSS PERFORMANCE \& RAP FORMULATION}

Based upon the imperfect repair model introduced in Section III, the average state distribution for each element $l$ can be calculated via (10), and it can be expressed by the UGF in a polynomial form as $u_{l}(z)=\sum_{i=1}^{k_{l}} \bar{p}_{(l, i)} \cdot z^{g_{(l, i)}}$; whereas the average system performance distribution can be also written in the same UGF fashion as $U_{s}(z)=\sum_{i=1}^{K_{s}} \bar{p}_{s i} \cdot z^{g_{s i}}$ which can be determined by UGFs of elements via the UGF composition rules [9]. The system availability in our work is defined as the probability that the system performance is not less than the user demand level [9], and it is formulated as

$$
A(W)=\sum_{j=1}^{H} q_{j} \sum_{i=1}^{K_{s}} \bar{p}_{s i} \cdot 1\left(g_{s i}-w_{j} \geq 0\right)
$$

where $H$ denotes possible user demand levels; $w_{j}$ is the $j^{\text {th }}$ possible user demand level, and $q_{j}$ is the associated probability.

Suppose a system has $M_{s u b}$ subsystems, and in each subsystem $i$ there are $V_{i}$ versions of elements available for use. Instead of the notation $l$ in the above sections, the subscript $i, j$ is used to denote that a version $j$ element is in subsystem $i$ in the subsequent context. The total expenditure for any element consists of two categories: the assigned repair cost $c_{(i, j)}^{f}$ for each element failure, and the replacement $\operatorname{cost} c_{(i, j)}^{r}$. It is reasonable to suppose $c_{(i, j)}^{r} \gg c_{(i, j)}^{f}$, because replacement oftentimes involves too much transportation and installation expenditure. The associated replacement policy for elements is thereby denoted as the replacement policy $N_{(i, j)}$. Hence, the total maintenance expenditure of element $(i, j)$ in its lifecycle is equal to $c_{(i, j)}^{f}\left(N_{(i, j)}-1\right)+c_{(i, j)}^{r}$. The average maintenance expenditure of element $(i, j)$ per unit time is given by

$\bar{c}_{(i, j)}=\frac{c_{(i, j)}^{f}\left(N_{(i, j)}-1\right)+c_{(i, j)}^{r}}{E\left(Y_{(i, j)}\left(N_{(i, j)}-1\right)\right)+E\left(X_{(i, j)}\left(N_{(i, j)}\right)\right)+E\left(Z_{(i, j)}\right)}$.

The overall average system maintenance expenditure per unit time is determined as

$$
\bar{C}_{s}=\sum_{i=1}^{M_{s u b}} \sum_{j=1}^{V_{i}} n_{(i, j)} \cdot \bar{c}_{(i, j)}
$$

where $n_{(i, j)}$ is the redundancy level of the version $j$ element in subsystem $i$.

With the purpose of achieving the required system availability $A_{0}$ under user demand, the RAP is to determine not only the optimal redundancy levels of elements of different versions, but also the optimal strategy of assigning repair $\operatorname{cost} c_{(i, j)}^{f *}$ and element replacement policy $N_{(i, j)}^{*}$ to minimize the average system expenditure within the lifecycle. It can be formulated as

$$
\begin{gathered}
\left\{n_{(1,1)}^{*}, \ldots, n_{\left(M_{s u b}, V_{M_{s u b}}\right)}^{*}, c_{(1,1)}^{f *}, \ldots, c_{\left(M_{s u b}, V_{M_{s u b}}\right)}^{f *}, N_{(1,1)}^{*},\right. \\
\left.\ldots, N_{\left(M_{s u b}, V_{M_{s u b}}\right)}^{*}\right\}=\underbrace{\arg }_{\substack{n_{(i, j)}, c_{(i, j)}^{f}, N_{(i, j)} \\
i \in\left\{1, \ldots, M_{s u b}\right\}, j \in\left\{1, \ldots, V_{i}\right\} \\
\text { s.t. } A(W) \geq A_{0}}}\left\{\bar{C}_{s} \rightarrow \min \right\} .
\end{gathered}
$$




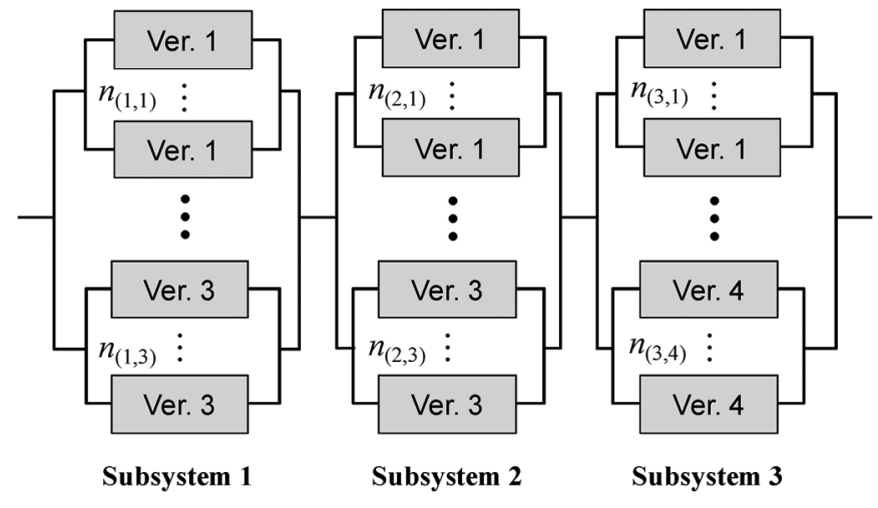

Fig. 2. The structure of a three-stage coal transportation system.

TABLE I

State Performance Rates, and Transition Intensities

\begin{tabular}{|c|c|c|c|c|c|c|}
\hline \multirow{2}{*}{ Subsystem } & \multirow{2}{*}{ Version } & \multicolumn{2}{|c|}{$\begin{array}{l}\text { State transition intensity } \\
\left(\mathrm{mon}^{-1}\right)\end{array}$} & \multicolumn{3}{|c|}{$\begin{array}{l}\text { State performance rate } \\
\text { (ton/day) }\end{array}$} \\
\hline & & $\lambda_{(2,1)}^{l}$ & $\lambda_{(3,2)}^{l}$ & $g_{(l, 1)}$ & $g_{(l, 2)}$ & $g_{(l, 3)}$ \\
\hline \multirow{3}{*}{1} & 1 & 0.10 & 0.08 & 0 & 30 & 60 \\
\hline & 2 & 0.12 & 0.08 & 0 & 60 & 100 \\
\hline & 3 & 0.10 & 0.06 & 0 & 80 & 130 \\
\hline \multirow{3}{*}{2} & 1 & 0.12 & 0.10 & 0 & 30 & 50 \\
\hline & 2 & 0.12 & 0.07 & 0 & 70 & 100 \\
\hline & 3 & 0.15 & 0.10 & 0 & 50 & 130 \\
\hline \multirow{4}{*}{3} & 1 & 0.10 & - & 0 & 40 & - \\
\hline & 2 & 0.15 & - & 0 & 70 & - \\
\hline & 3 & 0.12 & 0.08 & 0 & 60 & 120 \\
\hline & 4 & 0.12 & 0.10 & 0 & 80 & 150 \\
\hline
\end{tabular}

\section{Genetic Algorithm ImPlementation}

The optimization problem formulated in (22) is a complex non-linear optimization problem with mixed continuous and discrete decision variables. An exhaustive examination of all the solution space is not realistic due to the computational time constraint. The advanced meta-heuristic algorithms, such as genetic algorithm (GA), Tabu search, simulated annealing algorithm, and ant colony optimization (ACO), are effective approaches to search the global optimal solution in a computationally efficient manner. Among these algorithms, GA is one of the most widely used methods to solve various kinds of optimization problems due to its flexibility in representing both continuous and discrete decision variables, good global optimization capability, and it has been successfully applied to an abundance of optimization problems in reliability engineering (Levitin [9], and Tian et al. [18]), as well as maintenance optimization problems (Levitin and Lisnianski [15], [18], and Liu and Huang [33]). Thus, GA is tailored in this paper to facilitate searching the global optimal solution.

To apply the GA to a specific problem, solution representation is necessary. To keep the diversity of the population (a set of solutions), crossover, mutation, and selection procedures are executed during the searching iterations, with the solution referred to as a chromosome. The main GA procedure for the formulated optimization problem is as follows.

1. Population initialization. The chromosome consists of three parts with the length $L_{s}=\sum_{i=1}^{M_{s u b}} V_{i}$ for each part. Therefore, the entire length of the chromosome for a single
TABLE II

PARAMETERS OF EACH ELEMENT

\begin{tabular}{cccccccccc}
\hline \multirow{2}{*}{ Subsystem } & Version & $c_{l}^{r}$ & $c_{l}^{0}$ & $\alpha_{l}^{0}$ & $\xi_{l}$ & $\beta_{l}$ & $\mu_{Y_{1}^{l}}$ & $\mu_{Z_{l}}$ & $\zeta_{(l, 3)}$ \\
\hline \multirow{2}{*}{1} & 1 & 30 & 12 & 0.95 & 3 & 1.15 & 1 & 5 & 10 \\
& 2 & 45 & 20 & 0.9 & 3.5 & 1.2 & 1.5 & 7 & 12 \\
& 3 & 65 & 35 & 0.92 & 3.5 & 1.3 & 2 & 9 & 8 \\
\hline \multirow{3}{*}{2} & 1 & 30 & 15 & 0.94 & 5 & 1.1 & 0.8 & 6.5 & 9 \\
& 2 & 50 & 30 & 0.92 & 4 & 1.3 & 1.5 & 9 & 8.5 \\
& 3 & 65 & 38 & 0.9 & 5 & 1.2 & 2 & 10 & 11 \\
\hline \multirow{4}{*}{3} & 1 & 25 & 10 & 0.88 & 4.5 & 1.05 & 1 & 6 & - \\
& 2 & 30 & 15 & 0.9 & 4 & 1.1 & 2 & 8 & - \\
& 3 & 60 & 30 & 0.9 & 3 & 1.15 & 2.5 & 10 & 9 \\
& 4 & 72 & 40 & 0.93 & 4 & 1.3 & 3 & 12 & 10 \\
\hline
\end{tabular}

TABLE III

USER DEMAND LEVELS

\begin{tabular}{clllll}
\hline Demand (ton/day) & 120 & 100 & 80 & 60 & 30 \\
\hline Probability & 0.10 & 0.30 & 0.30 & 0.20 & 0.10 \\
\hline
\end{tabular}

solution is $3 L_{s}$, and the chromosome is denoted as a string $\mathbf{s}=\left\{s_{1}, \ldots, s_{L_{s}}, s_{L_{s}+1}, \ldots, s_{2 L_{s}}, s_{2 L_{s}+1}, \ldots, s_{3 L_{s}}\right\}$, where $s_{l}$ for $1 \leq l \leq L_{s}$ is the non-negative integer corresponding to redundancy level for the element of each version; $0<s_{l} \leq 1$ for $L_{s}+1 \leq l \leq 2 L_{s}$ represents the ratio of assigned repair cost to the maximum repair cost. Thus, the corresponding assigned repair cost is equal to $c_{l}^{f}=s_{l} \cdot c_{l}^{0}$; for $2 L_{s}+1 \leq l \leq 3 L_{s}, s_{l} \geq 1$ is the replacement policy for the element of each version. Then, one generates the initial population with size $N_{g}$.

2. Fitness evaluation. The average system expenditure $\bar{C}_{s}$ is used as the fitness value for each chromosome. To compute $\bar{C}_{s}$ and $A(W)$ for any individual chromosome, seven steps should be followed:

a. Derive the state probabilities $p_{(l, i)}(t)$ of the first repair cycle for each element via (1).

b. According to the assigned repair cost, compute $\alpha_{l}$, and $\mathbf{T}^{l}\left(\right.$ or $\mathbf{T}_{1}^{l}$ ) for each element via (15) through (18).

c. Derive the state probabilities $p_{(l, i)}^{n}(t), E\left(S_{(n, i)}^{l}\right)$, $E\left(X_{n}^{l}\right)$, and $E\left(Y_{n}^{l}\right)$ of remaining repair cycles for each element via (6) through (9).

d. Compute the average state probabilities $\bar{p}_{l, i}$ for each element via (10).

e. Generate the UFG $u_{l}(z)$ for each element based on the average state probabilities $\bar{p}_{l, i}$ derived from the last step.

f. Generate the UFG $U_{s}(z)$ for the entire systems by using UGFs of all the elements and the UGF composition rules.

g. Compute the average system maintenance expenditure $\bar{C}_{s}$, and the system availability $A(W)$ via (19) through (21).

The smaller $\bar{C}_{s}$, the higher the fitness value. The penalty function approach can be employed to handle infeasible solutions which violates the availability constraint $A(W) \geq A_{0}$. The first $N_{r}$ chromosomes with the highest fitness value in the current iteration are reserved for the next iteration.

3. New population generation. The roulette-wheel selection scheme is used to randomly select chromosomes from 
TABLE IV

Optimal SOLUTIONS IN DifFERENT CASES

\begin{tabular}{|c|c|c|c|c|c|c|c|c|c|c|}
\hline \multirow{2}{*}{ Subsystem } & \multirow[b]{2}{*}{ Version } & \multicolumn{3}{|c|}{ Case I } & \multirow{2}{*}{$\begin{array}{c}\text { Case II } \\
n_{(i, j)}^{*}\end{array}$} & \multicolumn{3}{|c|}{ Case III } & \multicolumn{2}{|c|}{ Case IV } \\
\hline & & $n_{(i, j)}^{*}$ & $c_{(i, j)}^{f^{*}}$ & $N_{(i, j)}^{*}$ & & $n_{(i, j)}^{*}$ & $c_{(i, j)}^{f}$ & $N_{(i, j)}^{*}$ & $n_{(i, j)}^{*}$ & $N_{(i, j)}^{*}$ \\
\hline \multirow{3}{*}{1} & 1 & 4 & 12.0 & 6 & - & - & - & - & 4 & 8 \\
\hline & 2 & - & - & - & 3 & - & - & - & - & - \\
\hline & 3 & - & - & - & - & 3 & 8.75 & 4 & - & - \\
\hline \multirow{3}{*}{2} & 1 & 3 & 3.1 & 4 & 1 & 1 & 3.75 & 1 & 1 & 5 \\
\hline & 2 & 1 & 14.9 & 3 & 3 & 3 & 7.5 & 4 & 2 & 3 \\
\hline & 3 & - & - & - & - & - & - & - & - & - \\
\hline \multirow{4}{*}{3} & 1 & - & - & - & - & - & - & - & 1 & 4 \\
\hline & 2 & - & - & - & - & - & - & - & - & - \\
\hline & 3 & 3 & 17.9 & 3 & 4 & - & - & - & 2 & 4 \\
\hline & 4 & - & - & - & - & 4 & 10 & 6 & - & - \\
\hline \multicolumn{2}{|c|}{$\bar{C}_{s}^{*}$} & \multicolumn{3}{|c|}{$10.80 \times 10^{5}$} & $18.54 \times 10^{5}$ & \multicolumn{3}{|c|}{$14.08 \times 10^{5}$} & \multicolumn{2}{|c|}{$11.22 \times 10^{5}$} \\
\hline \multicolumn{2}{|c|}{$A(W)$} & \multicolumn{3}{|c|}{0.8509} & 0.8503 & \multicolumn{3}{|c|}{0.8509} & \multicolumn{2}{|c|}{0.8514} \\
\hline
\end{tabular}

the current iteration based on their fitness values, to form a new population for the next iteration. The crossover and mutation operators are utilized to generate new chromosomes to keep the population diversity, and to explore the unsearched solution space. Because the chromosome consists of continuous, discrete digits with different range, the crossover and mutation operators are executed separately for each of the three parts to keep the digits within their allowable bounds. The $N_{r}$. chromosomes reserved in the previous iteration are put into the new generation directly.

4. Iterative process termination. The procedure terminates once the iteration count reaches $N_{c}$. Otherwise, go to Step 2 for the next iteration.

The computational efficiency of the GA is acceptable in our illustrative examples. If the computation time of the GA is unaffordable for systems consisting of a large number of components, many other advanced meta-heuristic algorithms with a greater computational efficiency can also be tailored to solve the resulting optimization problem.

\section{ILLUSTRATIVE EXAMPLE}

The studied system is a three-stage coal transportation system with a series-parallel structure, as shown in Fig. 2. For each subsystem, several versions of the elements are available to be chosen. The element state performance rates and the associated state transition intensities for the first repair cycle are tabulated in Table I. Other parameters related to maintenance time and cost, as well as characteristic parameters in the hypothetical (15) and (18), are listed in Table II, where the units for cost, and time are $\$ 10^{5}$, and one month, respectively.

Given the random user demand levels as shown in Table III, and the desired system availability $A_{0}=0.85$, the minimum average system maintenance expenditure per unit time can be achieved via optimally determining the redundancy levels, repair cost, and the replacement policy for each element version.

The proposed GA is executed 10 times via Matlab on a PC with an Intel Core(TM) Duo $2 \mathrm{GHz}$ CPU, and 4 GB RAM; and the mean, and standard deviation of computation time for a single run are $333.51 \mathrm{sec}$, and $77.94 \mathrm{sec}$ respectively, when $N_{g}$ is set to 80 , and $N_{c}$ is 500 . The best solution among these results is presented in Table IV as Case I. It shows that, except for element Version 1 in Subsystem 1, all elements of other versions are assigned a repair cost which is less than the maximum repair cost. For example, the optimal assigned repair $\operatorname{cost} c_{(i, j)}^{f *}$ for element Version 1 in Subsystem 2 is $\$ 3.1 \times 10^{5}$, which means the associated parameter $\alpha_{l}=0.6858$, and the probability matrix $\mathbf{T}_{1}^{l}(n)$ for this type of element can be expressed as

$$
\begin{aligned}
\mathbf{T}_{1}^{l}(n) & =\left[r_{(1,2)}^{l}(n), r_{(1,3)}^{l}(n)\right] \\
& =[0,1] \times\left[\begin{array}{cc}
1 & 0 \\
1-0.2067^{\left(\frac{1}{9.0}\right)} & 0.2067\left(\frac{1}{9.0}\right)
\end{array}\right]^{n} .
\end{aligned}
$$

Based on (6), the state probabilities of this type of element in each repair cycle can be written as

$p_{(l, i)}^{n}(t)=\sum_{j=i}^{3} r_{(1, j)}^{l}(n-1) \cdot p_{(l, i) \mid j}\left(0.6858^{1-n} t\right),(n=1,2,3,4$. $)$,

and they are plotted in Fig. 3. As shown in Fig. 3, compared to the previous repair cycle, the repaired element has a faster degradation trend in the next repair cycle. On the other hand, the probability that the repaired element is restored to the best state 3 decreases with the increase of the number of repair cycles, whereas the probability of being restored to state 2 increases.

To illustrate the validity of the analytical results from the proposed models, Monte Carlo simulation is used here, in which 10,000 realizations of deterioration processes of the examined element are randomly generated. It is expected that the analytical results should be identical with that of simulation. The flowchart for generating a realization of the deterioration process is illustrated in Fig. 4. For any individual realization of the examined element $l$, the amount of time $x_{(i, j)}$ that the element transits from any state $i$ with a higher performance rate to the state $j$ $(j \in\{i-1, i-2, \ldots, 1\})$ with a lower performance rate can be randomly generated by an exponential distribution based on the state transition intensities of the first repair cycle. In the $n^{t h}$ repair cycle, because the state transition intensities of the repaired element increase proportionally as proposed in this paper, the state transition time can be computed by $x_{(i, j)}^{\prime}=x_{(i, j)} \cdot \alpha_{l}^{n-1}$. The element will jump from the higher state $i$ into the lower state $k$ which has the minimal state transition time among other lower states, i.e. $x_{(i, k)}^{\prime}=\min \left\{x_{(i, j)}^{\prime}\right\}(j<i)$. The element will be repaired until it reaches the failure state, and restored to higher states with probability $\mathbf{T}_{1}^{l}(n)$. This simulation process continues running until the number of repairs reaches $N_{l}$; then an individual realization of the deterioration process of element $l$ is completely generated. In the $n^{t h}$ repair cycle, the state probability $p_{(l, i)}^{n}(t)$ at time $t$ equals the ratio of the number of elements being right at state $i$ to the total number of realizations. 

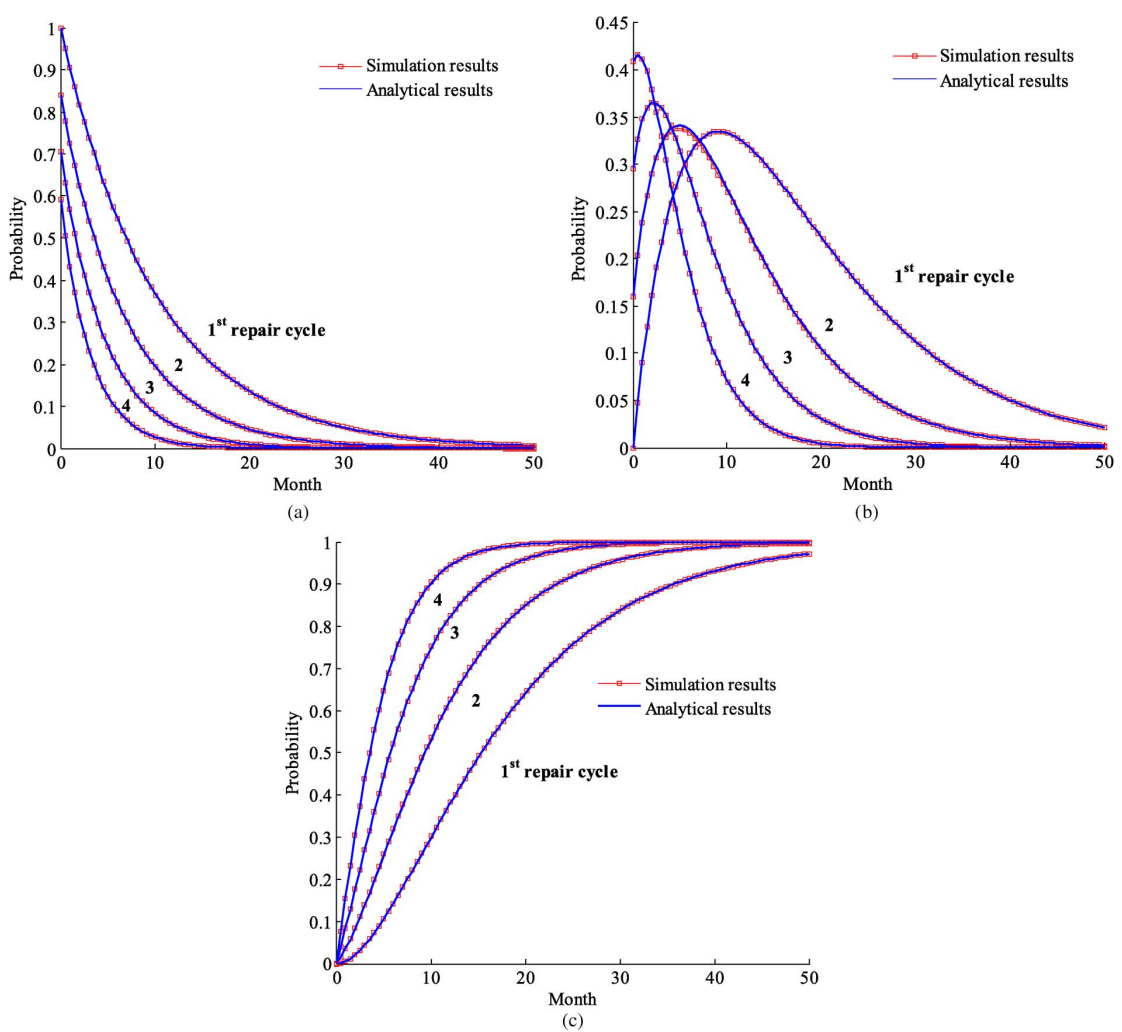

Fig. 3. The state probabilities from both analytical and simulation results for element Version 1 in Subsystem 2. (a) $p_{(l, 3)}^{n}(t)$ for state 3; (b) $p_{(l, 2)}^{n}(t)$ for state 2; (c) $p_{(l, 1)}^{n}(t)$ for state 1 .

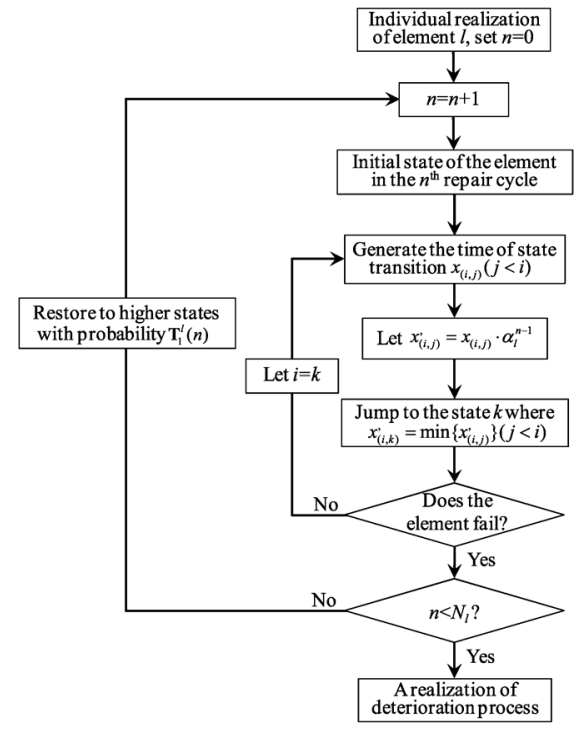

Fig. 4. The flowchart of generating a realization of the deterioration process.

As observed from Fig. 3, we can conclude that the results from the proposed models and simulation are identical.

To demonstrate the efficiency and effectiveness of the proposed method, solutions from other scenarios are tabulated in Table IV. Case II is the scenario where the failed elements are repaired as good as new (replacement) once they fail. In this Case II, the optimal system configuration is completely different from Case I, and the associated system expenditure is $72 \%$ higher than in Case I. It indicates that taking the imperfect mainte-
TABLE $\mathrm{V}$

Optimal Solutions Under VARIOUS ReQUiRed AVAILABILITY CONSTRAINTS

\begin{tabular}{|c|c|c|c|c|c|c|c|c|c|c|}
\hline \multirow{2}{*}{ Subsystem } & \multirow{2}{*}{ Version } & \multicolumn{3}{|c|}{$A_{0}=0.9$} & \multicolumn{3}{|c|}{$A_{0}=0.95$} & \multicolumn{3}{|c|}{$A_{0}=0.97$} \\
\hline & & $n_{(i, j)}^{*}$ & $c_{(i, j)}^{f^{*}}$ & $N_{(i, j)}^{*}$ & $n_{(i, j)}^{*}$ & $c_{(i, j)}^{f^{*}}$ & $N_{(i, j)}^{*}$ & $n_{(i, j)}^{*}$ & $c_{(i, j)}^{f^{*}}$ & $N_{(i, j)}^{*}$ \\
\hline \multirow{3}{*}{1} & 1 & 4 & 11.78 & 6 & 5 & 11.93 & 8 & 5 & 11.95 & 8 \\
\hline & 2 & - & - & - & - & - & - & - & - & - \\
\hline & 3 & - & - & - & - & - & - & - & - & - \\
\hline \multirow{3}{*}{2} & 1 & 5 & 3.89 & 4 & 3 & 2.72 & 5 & 1 & 8.63 & 4 \\
\hline & 2 & - & - & - & 2 & 5.43 & 3 & 3 & 15.45 & 3 \\
\hline & 3 & - & 24.0 & - & - & - & - & - & - & - \\
\hline \multirow{4}{*}{3} & 1 & 1 & 3.17 & 5 & 3 & 1.93 & 5 & 7 & 2.84 & 5 \\
\hline & 2 & - & - & - & - & - & - & - & - & - \\
\hline & 3 & 3 & 12.21 & 3 & 2 & 24.12 & 4 & - & - & - \\
\hline & 4 & - & - & - & - & - & - & - & - & - \\
\hline \multicolumn{2}{|c|}{$\bar{C}_{s}^{*}$} & \multicolumn{3}{|c|}{$11.96 \times 10^{5}$} & \multicolumn{3}{|c|}{$13.95 \times 10^{5}$} & \multicolumn{3}{|c|}{$14.94 \times 10^{5}$} \\
\hline \multicolumn{2}{|c|}{$A(W)$} & \multicolumn{3}{|c|}{0.9001} & \multicolumn{3}{|c|}{0.9500} & \multicolumn{3}{|c|}{0.9700} \\
\hline
\end{tabular}

nance efficiency into account will result a better solution than assuming the maintenance is perfect. With the aim to show the advantage of assigning the repair cost for each element strategically over distributing evenly, the optimal solution of Case III where the ratio $c_{i, j}^{f} / c_{i, j}^{0}$ is identical for each version of the elements is also listed in Table IV. The optimal value of the ratio $c_{i, j}^{f} / c_{i, j}^{0}$ solved by GA optimization is 0.25 . Obviously, for Case III, the minimum achievable system expenditure is somewhat higher, with percentages of $30 \%$, than the Case I, but is lower than Case II. Case IV is the situation of assigning a maximum repair cost (i.e. $c_{l}^{0}$ ) to each repair actions, and the corresponding system expenditure is $4 \%$ higher than in Case I. We conclude that strategically assigning the repair cost for each element according to their repair cost versus repair efficiency relation is more beneficial than evenly or maximally distributing cases. 
To study the impact from the availability constraint, we tabulate the optimal solutions under different desired availability in Table V. As the required availability becomes higher, the average system expenditure increases, and the system configurations vary from case to case. The number of Version 2 elements in Subsystem 2 increases as the required availability changes from 0.9 to 0.97 ; whereas the number of Version 1 elements decreases. The Version 2 element is more cost efficient compared to Version 1, with the increase of the desired availability. The similar observation can also be found for Subsystem 3, where the Version 1 element outperforms others in the cases with a higher availability constraint.

\section{REMARKS AND CLOSURE}

This paper develops a RAP considering a joint optimization of the redundancy level and maintenance strategy in the system design stage. Compared to the existing literature that accounts for the same issues, the unique features of this paper are twofold. First, nearly all the existing literature assumes that repaired elements are either in as good as new condition, essentially replaced at failure, or in as bad as old condition, essentially unchanged at failure. It has been argued that this hypothesis cannot hold in most practical problems. To quantify the imperfect maintenance efficiency, a generalized imperfect repair model is proposed in this paper to characterize the monotonic increasing trend of the element deteriorating process after repair. The essential characteristics of the proposed model are that, not only will the transition pace of the repaired element proportionally increase in the next repair cycle, but also the probability of the states in which the repaired element restores to will vary as the repair number increases. The extended imperfect maintenance model is more general, and has a broader application than the one proposed in our previous work. The second important feature of this work is intended to link the assigned repair cost with its corresponding repair efficiency to allow a more economically efficient maintenance management for MSSs. Two hypothetical functions are proposed to construct the functional relationship between the parameters of imperfect repair models and the assigned repair cost. The proposed model provides the flexibility to determine the system configuration along with the maintenance strategy at the early system design phase to achieve the desired system availability with minimum expected lifecycle expenditure.

It is noteworthy that, even though the proposed method is only demonstrated via a specific scenario where elements are repaired only when they fall into failure state, it can be further extended to a more general case where the preventive maintenance activities are conducted at immediate states of elements if the transition probability matrix $\mathbf{T}^{l}(n)$ is completely known in advance. The generalized imperfect repair model developed in this paper provides an alternative tool to quantify the imperfect maintenance efficiency for MSEs, and it is able to capture the phenomena that the degradation pace of repaired elements will proportionally increase with the times of repairs. However, note that not all the repairable MSSs possess this feature in practice. Model validation or selection is, therefore, required before applying this model to a specific engineered system [48]. Meanwhile, there is still a need for developing more imperfect maintenance models with various underlying physics to accurately characterize complex stochastic behaviors of repairable MSSs.

\section{REFERENCES}

[1] W. Kuo and R. Wan, "Recent advances in optimal reliability allocation," IEEE Trans. Syst. Man Cybern. A, vol. 77, no. 1, pp. 143-156, 2007.

[2] W. Kuo, H. Lin, Z. Xu, and W. Zhang, "Reliability optimization with the Lagrange multiplier and branch-and-bound technique," IEEE Trans. Rel., vol. 36, no. 5, pp. 624-630, 1987.

[3] C. S. Sung and Y. K. Cho, "Branch-and-bound redundancy optimization for a series system with multiple-choice constraints," IEEE Trans. Rel., vol. 48, no. 2, pp. 108-117, Jun. 1999.

[4] C. H. Ha and W. Kuo, "Multi-path approach for reliability-redundancy allocation using a scaling method," J. Heuristics, vol. 11, no. 3, pp. 201-217, 2005.

[5] D. W. Coit and A. E. Smith, "Reliability optimization of series-parallel systems using a genetic algorithm," IEEE Trans. Rel., vol. 45, no. 2, pp. 254-260, Jun. 1996.

[6] Y. C. Liang and A. E. Smith, "An ant colony optimization algorithm for the redundancy allocation problem (RAP)," IEEE Trans. Rel., vol. 53, no. 3, pp. 417-423, Sep. 2004.

[7] S. Kulturel-Konak, A. E. Smith, and D. W. Coit, "Efficiently solving the redundancy allocation problem using tabu search," IIE Trans., vol. 35 , no. 6 , pp. 515-526, 2003.

[8] B. Suman, "Simulated annealing-based multiobjective algorithms and their application for system reliability," Eng. Optimiz., vol. 35, no. 4, pp. 391-416, 2003.

[9] A. Lisnianski and G. Levitin, Multi-State Syst. Rel. Assessment, Optimiz., Appl.. Singapore: World Scientific, 2003.

[10] G. Levitin, A. Lisnianski, H. Ben-Haim, and D. Elmakis, "Redundancy optimization for series-parallel multi-state systems," IEEE Trans. Rel., vol. 47, no. 2, pp. 165-172, Jun. 1998.

[11] M. Agarwal and R. Gupta, "Homogeneous redundancy optimization in multi-state series-parallel systems: A heuristic approach," IIE Trans., vol. 39 , no. 3, pp. $277-289,2007$

[12] J. E. Ramirez-Marquez and D. W. Coit, "A heuristic for solving the redundancy allocation problem for multi-state series-parallel systems," Rel. Eng. Syst. Safety, vol. 83, no. 3, pp. 341-349, 2004.

[13] Z. Tian, M. J. Zuo, and H. Z. Huang, "Reliability-redundancy allocation for multi-state series-parallel systems," IEEE Trans. Rel., vol. 57, no. 2, pp. 303-310, Jun. 2008

[14] U. K. D. Kumar, J. E. Ramırez-Marquez, D. Nowicki, and D. Verma, "Reliability and maintainability allocation to minimize total cost of ownership in a series-parallel system," J. Risk Rel., vol. 221, no. 2, pp. 133-140, 2007.

[15] G. Levitin and A. Lisnianski, "Joint redundancy and maintenance optimization for multi-state series-parallel systems," Rel. Eng. Syst. Safety, vol. 64, no. 1, pp. 33-42, 1999.

[16] M. Nourelfath and Y. Dutuit, "A combined approach to solve the redundancy optimization problem for multi-state systems under repair policies," Rel. Eng. Syst. Safety, vol. 86, no. 3, pp. 205-213, 2004.

[17] M. Nourelfath and D. Ait-Kadi, "Optimization of series-parallel multistate systems under maintenance policies," Rel. Eng. Syst. Safety, vol. 92, no. 12, pp. 1620-1626, 2007.

[18] Z. Tian, G. Levitin, and M. J. Zuo, "A joint reliability-redundancy optimization approach for multi-state series-parallel systems," Rel. Eng. Syst. Safety, vol. 94, no. 10, pp. 1568-1576, 2009.

[19] H. Pham and H. Z. Wang, "Imperfect maintenance," Eur. J. Oper. Res., vol. 94 , no. 3, pp. 425-438, 1996

[20] T. Nakagawa, "Optimum policies when preventive maintenance is imperfect," IEEE Trans. Rel., vol. 28, no. 4, pp. 331-332, Dec. 1979.

[21] H. W. Block, W. S. Borges, and T. H. Savits, "Age dependent minimal repair,” J. Appl. Probab., vol. 22, no. 2, pp. 370-385, 1985.

[22] V. Makis and A. K. S. Jardine, "Optimal replacement policy for a general model with imperfect repair," J. Oper. Res. Soc., vol. 42, no. 2, pp. 111-120, 1992.

[23] M. Kijima, H. Morimura, and Y. Suzuki, "Periodical replacement problem without assuming minimal repair," Eur. J. Oper. Res., vol. 37, no. 2, pp. 194-203, 1988 .

[24] M. Kijima, "Some results for repairable systems with general repair," J. Appl. Probab., vol. 26, no. 1, pp. 89-102, 1989.

[25] M. A. K. Malik, "Reliable preventive maintenance policy," AIIE Trans., vol. 11, no. 3, pp. 221-228, 1979.

[26] D. Lin, M. J. Zuo, and R. C. M. Yam, "General sequential imperfect preventive maintenance models," Int. J. Rel., Qual. Safety Eng., vol. 7 , no. 3, pp. 253-266, 2000.

[27] Y. Lam, "A note on the optimal replacement problem," Adv. Appl. Probab., vol. 20, no. 2, pp. 479-482, 1988. 
[28] H. Z. Wang and H. Pham, "A quasi renewal process and its application in the imperfect maintenance," Int. J. Syst. Sci., vol. 27, no. 10, pp. 1055-1062, 1996

[29] H. Z. Wang and H. Pham, Rel. Optimal Maintenance. Berlin, Germany: Springer-Verlag, 2006.

[30] S. Wu and M. J. Zuo, "Linear and nonlinear preventive maintenance models," IEEE Trans. Rel., vol. 59, no. 1, pp. 242-249, Mar. 2010.

[31] G. Levitin and A. Lisnianski, "Optimization of imperfect preventive maintenance for multi-state systems," Rel. Eng. Syst. Safety, vol. 67, no. 2, pp. 193-203, 2000.

[32] N. Nahas, A. Khatab, D. Ait-Kadi, and M. Nourelfath, "Extended great deluge algorithm for the imperfect preventive maintenance optimization of multi-state systems," Rel. Eng. Syst. Safety, vol. 93, no. 11, pp. $1658-1672,2008$.

[33] Y. Liu and H. Z. Huang, "Optimal selective maintenance strategy for multi-state systems under imperfect maintenance," IEEE Trans. Rel., vol. 59, no. 2, pp. 356-367, Jun. 2010.

[34] I. W. Soro, M. Nourelfath, and D. Ait-Kadi, "Performance evaluation of multi-state degraded systems with minimal repairs and imperfect preventive maintenance," Rel. Eng. Syst. Safety, vol. 95, no. 2, pp. 65-69, 2010.

[35] C. M. Tan and N. Raghavan, "A framework to practical predictive maintenance modeling for multi-state systems," Rel. Eng. Syst. Safety, vol. 93, no. 8, pp. 1138-1150, 2008.

[36] Y. Liu and H. Z. Huang, "Comment on "A framework to practical predictive maintenance modeling for multi-state systems" by Tan C. M. and Raghavan N. [Reliab Eng Syst Saf 2008; 93(8):1138-50]," Rel. Eng. Syst. Safety, vol. 94, no. 3, pp. 776-780, 2009.

[37] M. Nourelfath, E. Châtelet, and N. Nahas, "Joint redundancy and imperfect preventive maintenance optimization for series-parallel multistate degraded systems," Rel. Eng. Syst. Safety, vol. 103, pp. 51-60, 2012.

[38] Y. Liu and H. Z. Huang, "Optimal replacement policy for multi-state system under imperfect maintenance," IEEE Trans. Rel., vol. 42, no. 10, pp. 734-745, 2010.

[39] A. Lisnianski, "Estimation of boundary points for continuum-state system reliability measures," Rel. Eng. Syst. Safety, vol. 74, no. 1, pp. 81-88, 2001.

[40] Y. Liu, H. Z. Huang, and G. Levitin, "Reliability and performance assessment for fuzzy multi-state element," J. Risk Rel., vol. 222, no. 4, pp. 675-686, 2008.

[41] Y. Liu and H. Z. Huang, "Reliability assessment for fuzzy multi-state system," Int. J. Syst. Sci., vol. 41, no. 4, pp. 365-379, 2010.

[42] A. Lisnianski, "Extended block diagram method for a multi-state system reliability assessment," Rel. Eng. Syst. Safety, vol. 92, no. 12, pp. 1601-1607, 2007.

[43] K. Trivedi, Probability and Statistics With Reliability, Queuing and Computer Science Application. Hoboken, NJ, USA: Wiley, 2002.

[44] G. Levitin, T. Zhang, and M. Xie, "State probability of a series-parallel repairable system with two-types of failure states," Int. J. Syst. Sci., vol. 37, no. 14-15, pp. 1011-1020, 2006.

[45] W. Li, "Incorporating aging failures in power system reliability evaluation," IEEE Trans. Power Syst., vol. 17, no. 3, pp. 918-923, Mar. 2002.

[46] C. H. Lie and Y. H. Chun, "An algorithm for preventive maintenance policy," IEEE Trans. Rel., vol. 35, no. 1, pp. 71-75, Mar. 1986.

[47] M. A. K. Malik, "Reliable preventive maintenance scheduling," IIE Trans, vol. 11, no. 3, pp. 221-228, 1979.

[48] Y. Liu, H. Z. Huang, and X. Zhang, "A data-driven approach to selecting imperfect maintenance models," IEEE Trans. Rel., vol. 61, no. 1, pp. 101-112, Mar. 2012.
Yu Liu is an Associate Professor in the School of Mechanical, Electronic, and Industrial Engineering, at the University of Electronic Science and Technology of China. He received his Ph.D. degree in Mechatronics Engineering from the University of Electronic Science and Technology of China. He was a visiting pre-doctoral fellow in the Department of Mechanical Engineering at Northwestern University, Evanston, U.S.A. from 2008 to 2010. His research interests include system reliability modeling and analysis, maintenance decisions, prognostics and health management, and design under uncertainty.

Hong-Zhong Huang is a Professor and the Dean of the School of Mechanical, Electronic, and Industrial Engineering, at the University of Electronic Science and Technology of China. He has held visiting appointments at several universities in the USA, Canada, and Asia. He received a Ph.D. degree in Reliability Engineering from Shanghai Jiaotong University, China, and has published 150 journal papers and 5 books in the fields of reliability engineering, optimization design, fuzzy sets theory, and product development. He is a Fellow of ISEAM (International Society of Engineering Asset Management), and a member of the ESRA (European Safety and Reliability Association) Technical Committee on System Reliability, a Regional Editor of International Journal of Reliability and Applications, an Editorial Board Member of International Journal of Reliability, Quality and Safety Engineering, International Journal of Quality, Statistics, and Reliability, International Journal of Reliability and Quality Performance. He received the William A. J. Golomski Award from the Institute of Industrial Engineers in 2006, the Best Paper Award of the 8th International Conference on Frontiers of Design and Manufacturing in 2008, and the Best Paper Award of the International Conference on Materials and Reliability in 2011. His current research interests include system reliability analysis, warranty, maintenance planning and optimization, and computational intelligence in product design.

Zhonglai Wang received the Ph.D. degree in Mechatronics Engineering from the University of Electronic Science and Technology of China. He is currently an Associate Professor in the School of Mechanical, Electronic, and Industrial Engineering at the University of Electronic Science and Technology of China. He has been a visiting scholar in the Department of Mechanical and Aerospace Engineering, Missouri University of Science and Technology from 2007 to 2008 . His research interests include reliability-based design, and robust design.

Yanfeng $\mathbf{L i}$ is currently a Ph.D. candidate in Mechatronics Engineering at the University of Electronic Science and Technology of China. His research interests include reliability analysis and evaluation of complex systems, dynamic fault tree modeling, Bayesian networks modeling, and probabilistic inference.

Yuanjian Yang is currently a Ph.D. candidate in Mechatronics Engineering at the University of Electronic Science and Technology of China. His research interests include reliability testing, reliability analysis and evaluation of complex systems, and dynamic fault tree modeling. 